\title{
Supplementary Materials for Magnesium calcium phosphate cement incorporating citrate for vascularized bone regeneration
}

Xiaopei Wu, ${ }^{\dagger}+$ Honglian Dai, $*, \dagger$ Suchun Yu, ${ }^{\dagger}$ Yanan Zhao, ${ }^{\dagger}$ Yanpiao Long, ${ }^{\dagger}$ Wenqin $\mathrm{Li},{ }^{\dagger}$ and Jing $\mathrm{Tu}^{\dagger}$

$†$ State Key Laboratory of Advanced Technology for Materials Synthesis and Processing, Biomedical Materials and Engineering Research Center of Hubei Province, Wuhan University of Technology, Wuhan 430070, China

†Foshan Xianhu Laboratory of the Advanced Energy Science and Technology Guangdong Laboratory, Xianhu hydrogen Valley, Foshan 528200, China

*Corresponding address: State Key Laboratory of Advanced Technology for Materials Synthesis and Processing, Wuhan University of Technology, Wuhan, 430070, PR China.

E-mail: daihonglian@whut.edu.cn

This file includes:

- Fig. S1. Characterization of different MCPC ${ }^{[1]}$.

- $\quad$ Fig. S2. Quantitative analysis of the new bone volume after 4, 8 weeks of surgery by micro-CT.

- Fig. S3. HE staining and Masson trichrome staining of new bone formation.

- Fig. S4. Immunohistochemistry staining of angiogenesis and osteogenesis in the defect area at 8 weeks.

- $\quad$ Fig. S5. VEGF in KEGG Pathway.

- $\quad$ Fig. S6. TGF- $\beta$ in KEGG Pathway.

- Fig. S7. HIF-1 $\alpha$ in KEGG Pathway.

- $\quad$ Fig. S8. bFGF in KEGG Pathway.

Number of pages: 6

Number of figures: 8

Number of tables: 0 
A

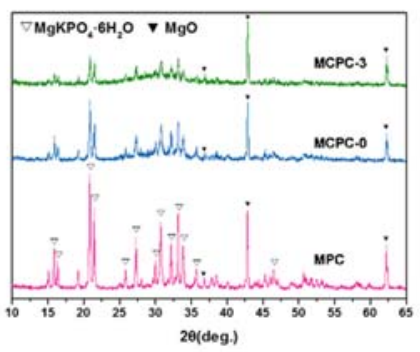

D

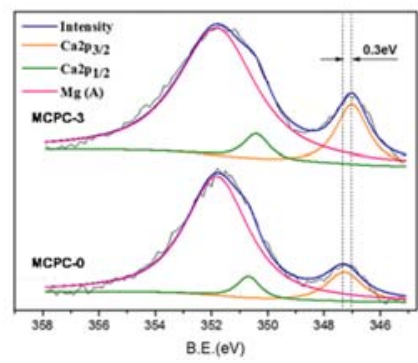

B

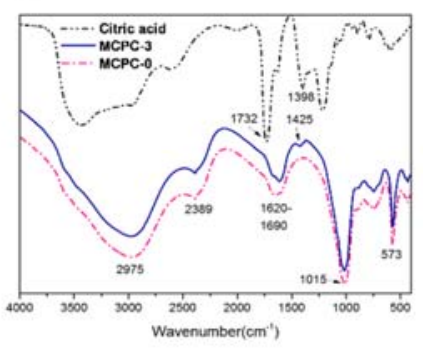

C

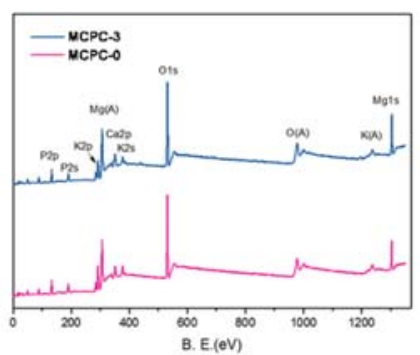

E

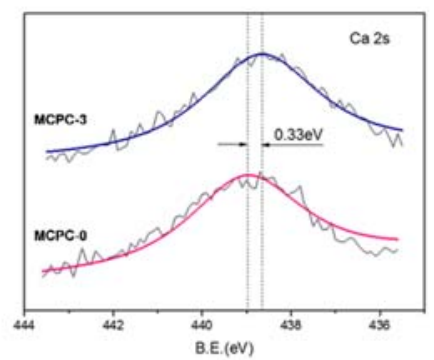

Fig. S1. Characterization of different MCPC ${ }^{[1]}$. (A) XRD patterns of MCPC after curing for 6 min under $37^{\circ} \mathrm{C}, 100 \%$ humidity. (B) FT-IR spectra of hardened MCPC-0, MCPC-3 and citric acid. (C) The full XPS spectra, (D) Ca2p spectra, (E) Ca2s spectra of hardened MCPC-0 and MCPC-3.

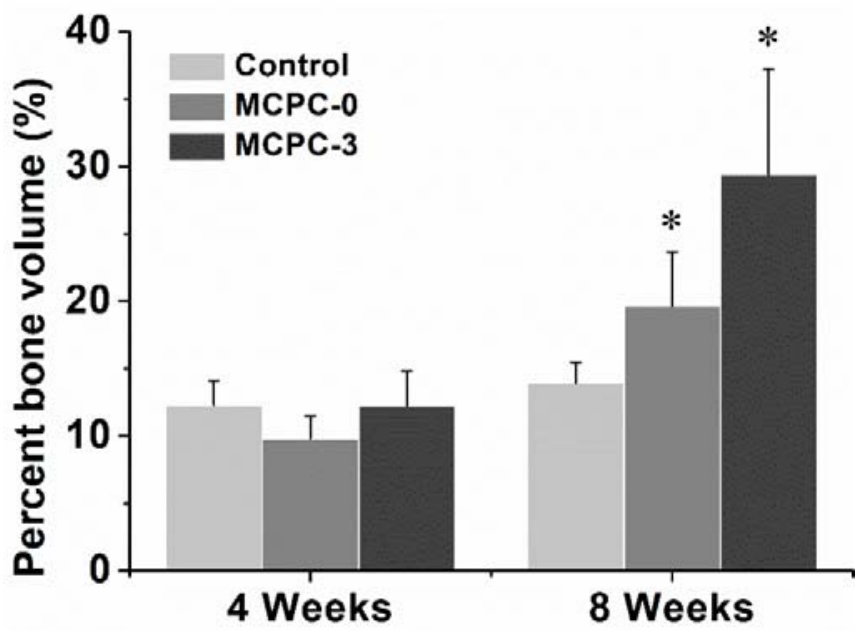

Fig. S2. Quantitative analysis of the new bone volume after 4,8 weeks of surgery by microCT. 


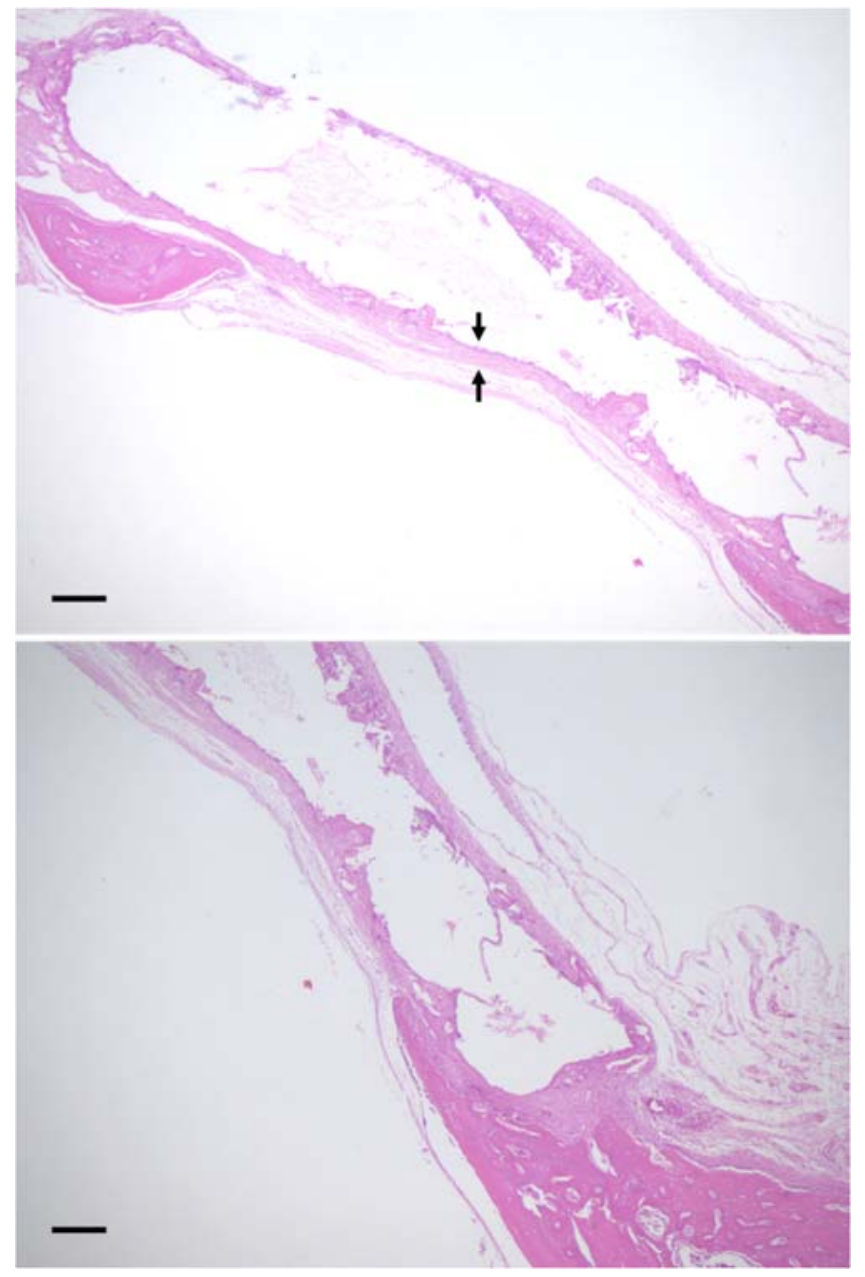

Fig. S3. HE staining and Masson trichrome staining of new bone formation. There are few new bone tissues and blood vessels in the defect area at 8 weeks. Scale bars $=200 \mu \mathrm{m}$. 


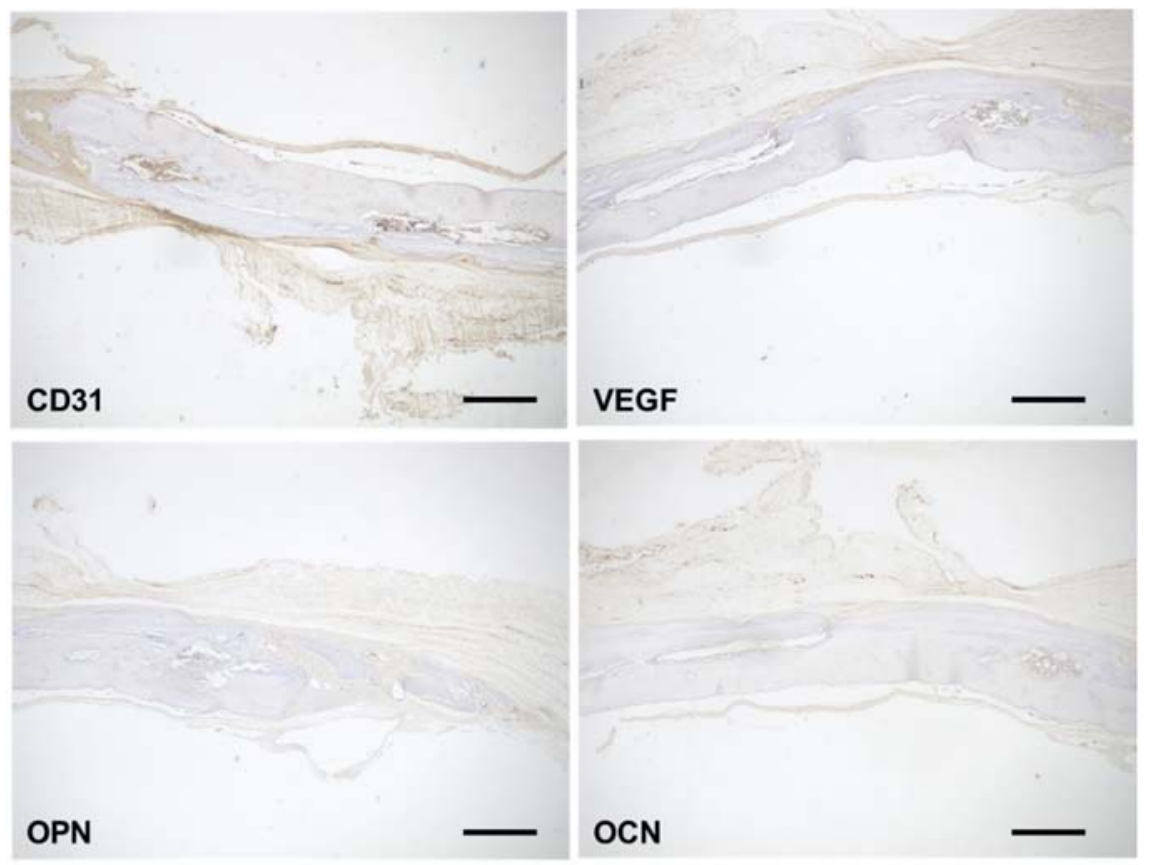

Fig. S4. Immunohistochemistry staining of angiogenesis and osteogenesis in the defect area at 8 weeks. Scale bars $=400 \mu \mathrm{m}$.

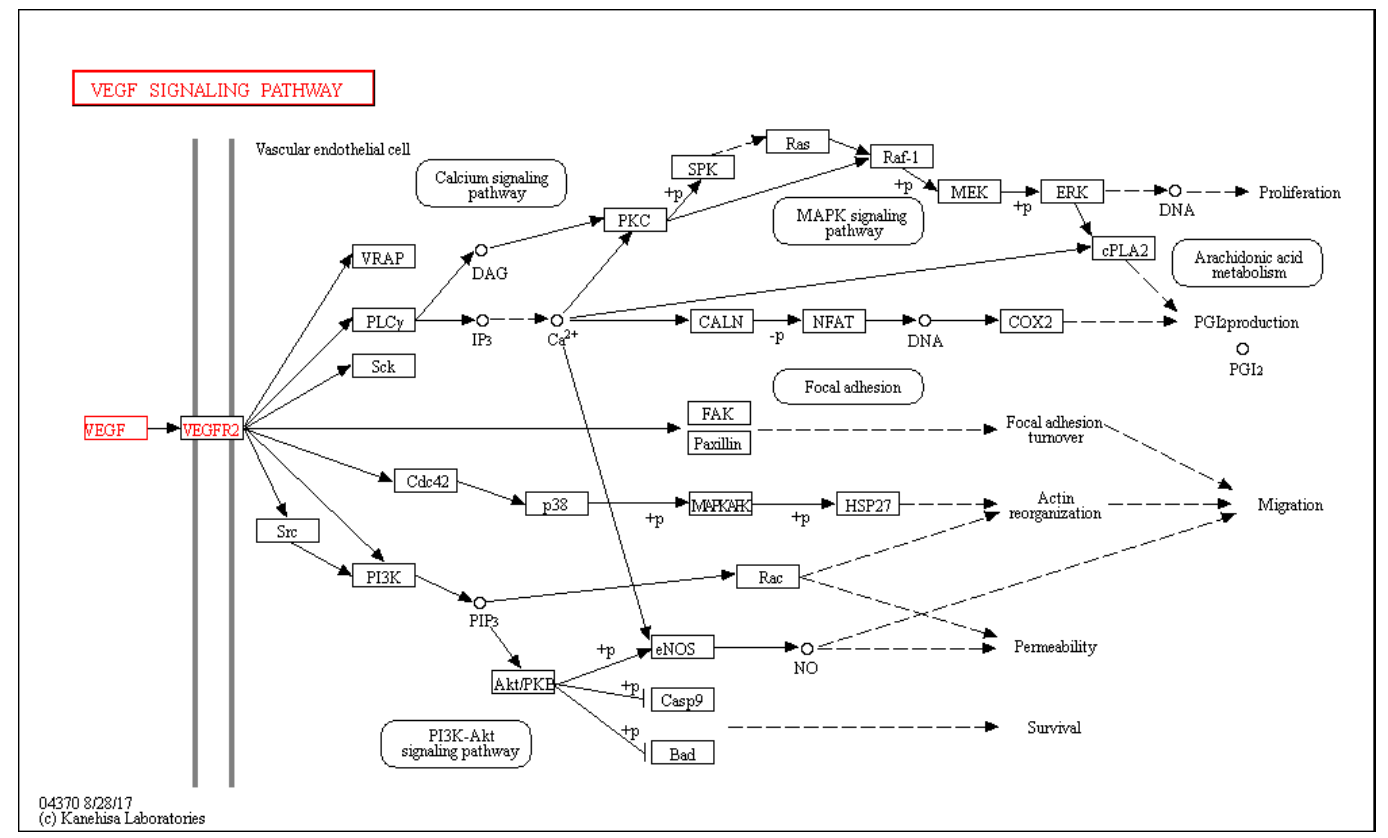

Fig. S5. VEGF in KEGG Pathway. 


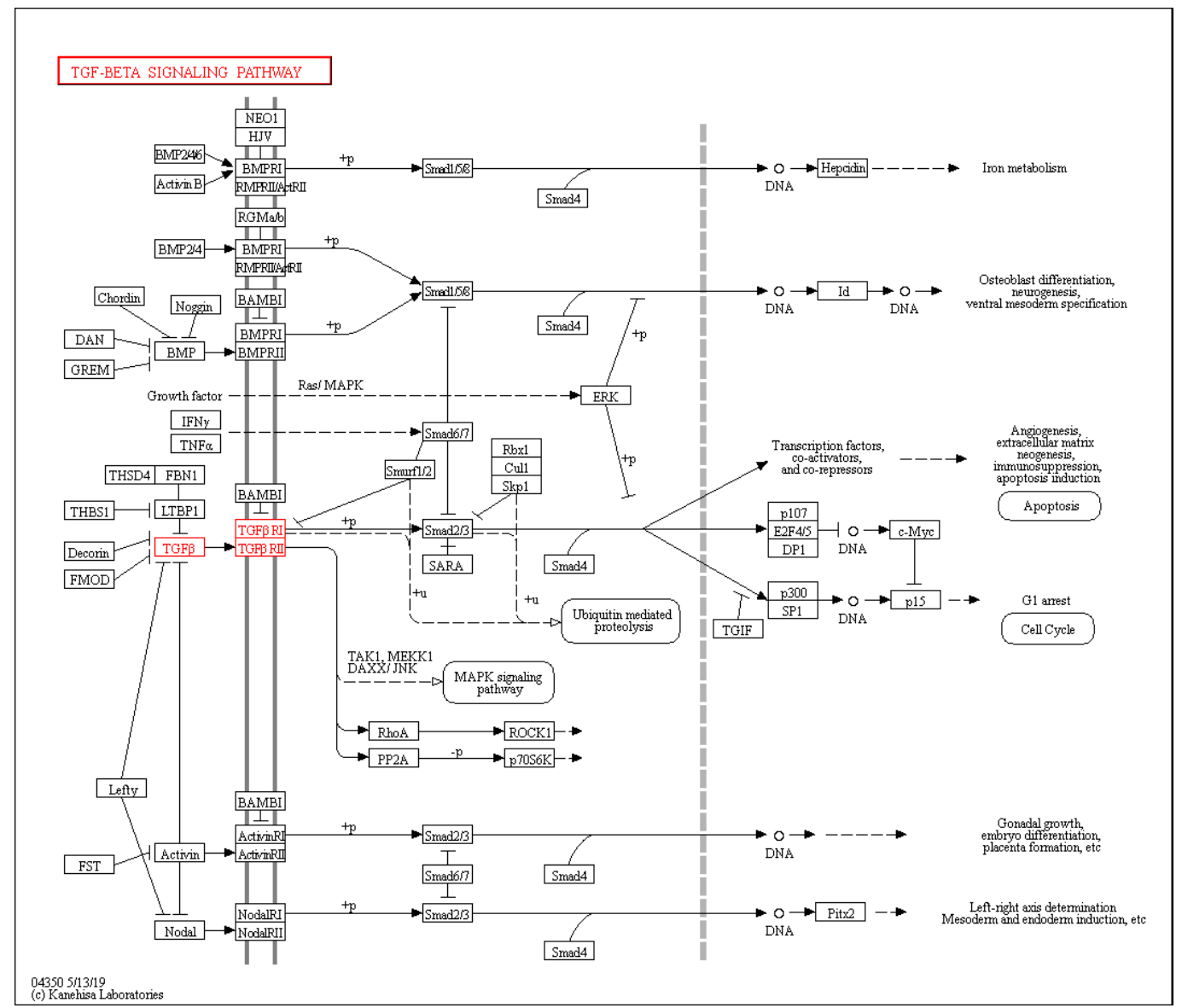

Fig. S6. TGF- $\beta$ in KEGG Pathway.

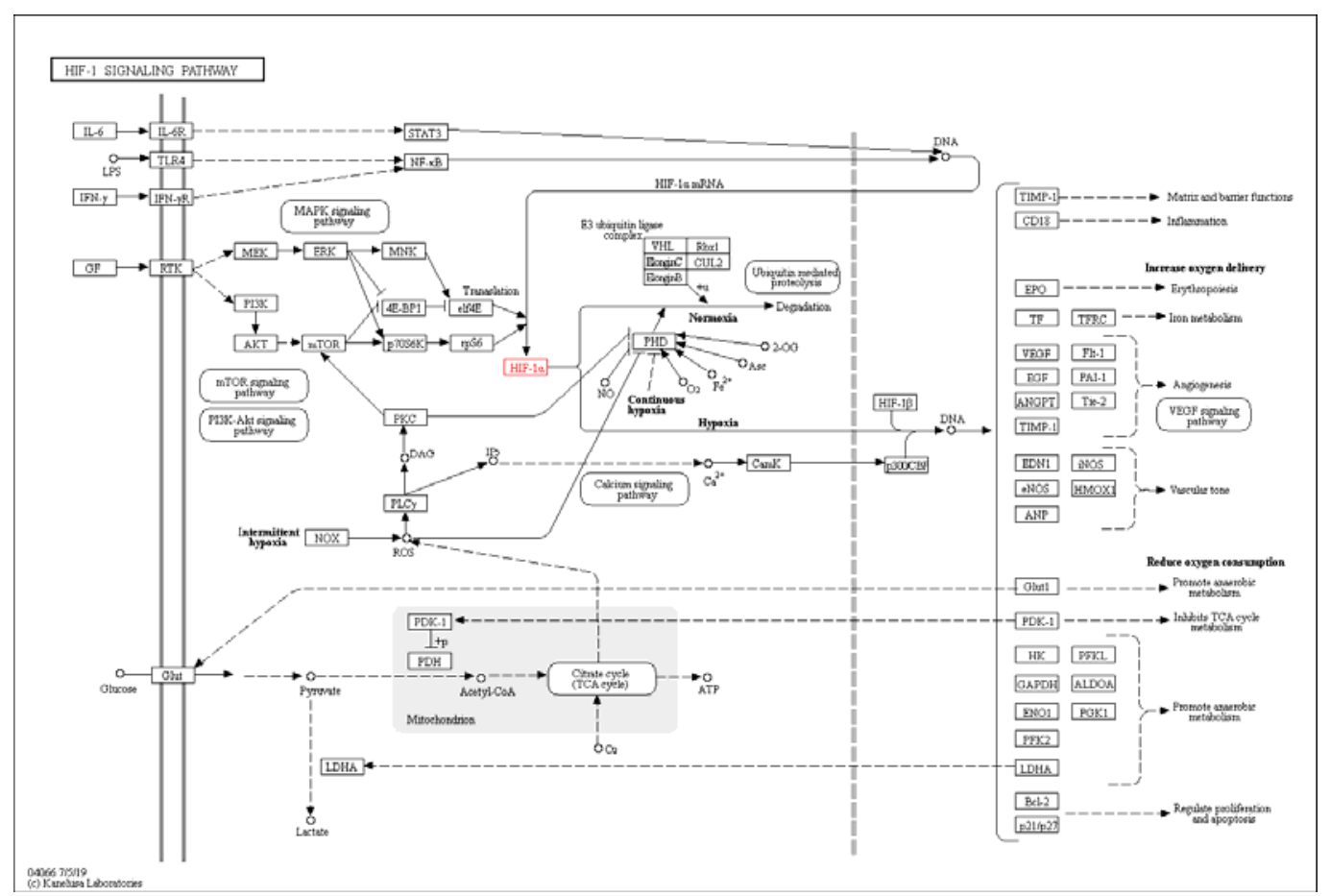

Fig. S7. HIF-1 a in KEGG Pathway. 


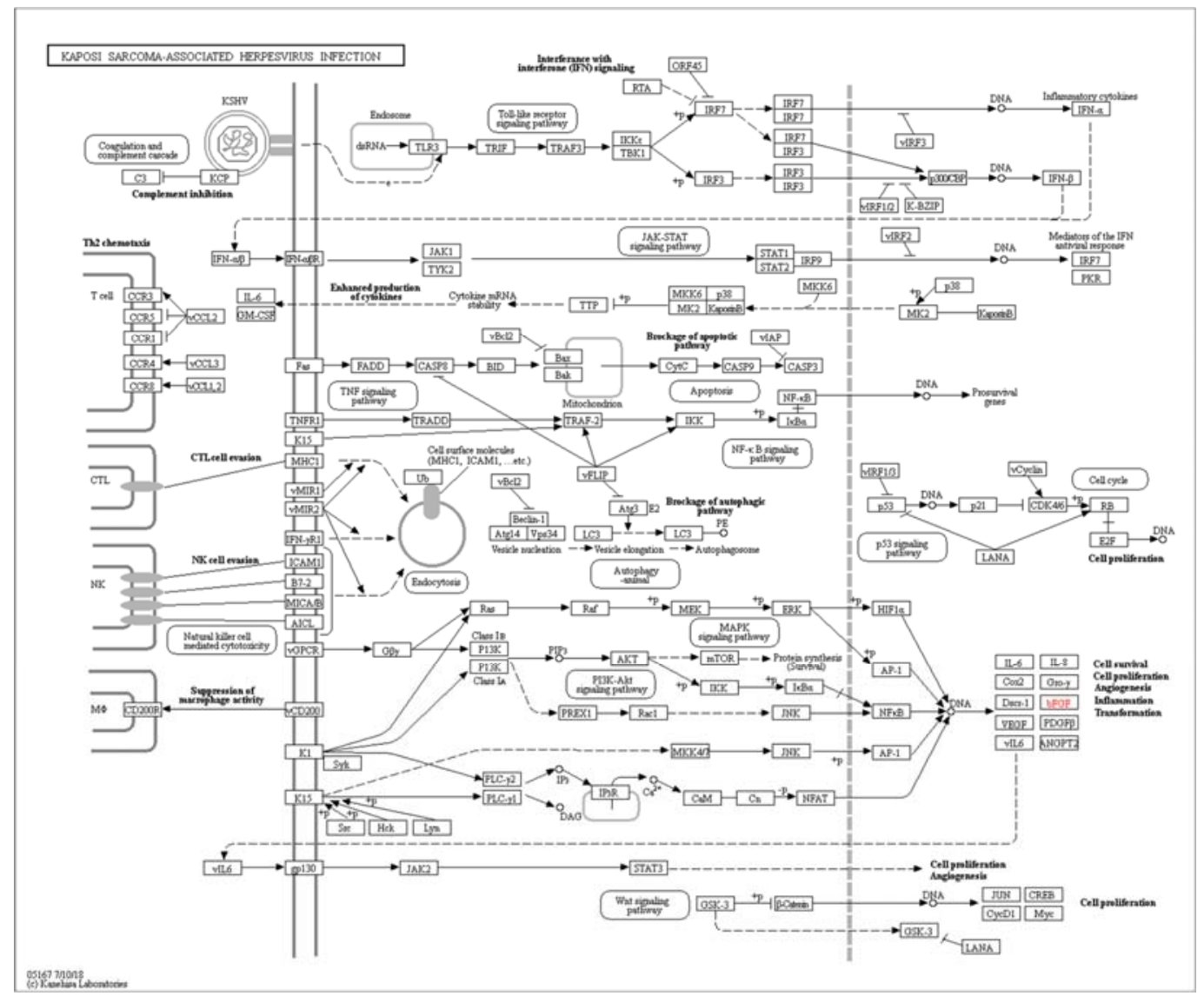

Fig. S8. bFGF in KEGG Pathway.

\section{References}

[1] Yu S, Liu L, Xu C, Dai H L. Magnesium phosphate-based cement with improved setting, strength and cytocompatibility properties by adding $\mathrm{Ca}(\mathrm{H} 2 \mathrm{PO} 4) 2 \cdot \mathrm{H} 2 \mathrm{O}$ and citric acid. Journal of the mechanical behavior of biomedical materials, 2019, 91: 229236. 